\title{
Correlation and Path Coefficient Analysis in Tomato (Solanum lycopersicum L.)
}

\author{
K. Sushma ${ }^{1 *}$, P. Saidaiah ${ }^{1}$, K. Ravinder Reddy ${ }^{2}$, Harikishan Sudini ${ }^{3}$ and A. Geetha ${ }^{4}$ \\ ${ }^{1}$ Department of Vegetable Science, ${ }^{1}$ Department of Genetics and Plant Breeding, ${ }^{2}$ Department \\ of Vegetable Science, College of Horticulture, SKLTSHU, Hyderabad, India \\ ${ }^{3}$ Department of Plant Pathology, ICRISAT, Patancheru, Telangana, India \\ ${ }^{4}$ Department of Crop Physiology, College of Agriculture, PJTSAU, Hyderabad, India
}

*Corresponding author

\section{A B S T R A C T}

\section{Keywords}

Tomato, Solanum lycopersicum, Correlation, Path coefficient, Fruit yield

\section{Article Info}

Accepted:

20 October 2020

Available Online:

10 November 2020
Correlation and path analysis were carried out in twenty three tomato genotypes for yield characters. The correlation coefficient studies showed that positive association of fruit yield per plant with plant height, number of primary branches per plant, number of fruits per plant, days to last harvest, fruit length, fruit width, average fruit weight, ascorbic acid, total soluble solids and lycopene content. Path coefficient showed that number of fruits per plant, average fruit weight, fruit width, ascorbic acid, total soluble solids and lycopene content had high positive direct effects on fruit yield per plant. Hence, these characters should be given more helpful in selection programme of high yielding genotypes in tomato.

\section{Introduction}

Tomato is a self-pollinated crop with chromosome number of $2 \mathrm{n}=24$ and belongs to the family Solanaceae. The cultivated tomato originated in a wild form in the PeruEcuador-Bolvia area of the Andes (South American) (Vavilov, 1951). Tomato is a rich source of vitamins (A and $\mathrm{C}$ ), minerals ( $\mathrm{Ca}$, $\mathrm{P}$ and $\mathrm{Fe}$ ), consumption of tomato and processed products has been associated with lower risk of developing digestive tract and prostrate cancer (Giovannucci et al., 2002). Correlation studies between fruit weight and its components and their relative contribution to yield are of value in planning a breeding program. Path analysis facilitates the partitioning of the correlation coefficient into direct and indirect effects on yield and any other attributes (Islam et al., 2010). Hence, keeping the above in view the present investigation was carried out to 
study correlation and path coefficient analysis.

\section{Materials and Methods}

The present study was carried out at the PG Research Block, Department of Vegetable Science, College of Horticulture, Rajendranagar, Sri Konda Laxman Telangana State Horticultural University, Hyderabad, which comes under sub-tropical zone and farm is situated at a latitude of $17.19^{\circ} \mathrm{N}$, longitude of $79.23^{\circ} \mathrm{E}$ and a altitude of $542.3 \mathrm{~m}$ above mean sea level. The seeds of twenty three genotypes were raised in protrays in College of Horticulture, Rajendranagar during second week of July, 2018. Twenty five days old seedlings were transplanted in the second week of August, 2018 in the main field. The experiment was laid out with twenty three genotypes of tomato in Randomized Block Design (RBD) with three replications (Table 1). Each germplasm line was grown in a plot of 1.8 $\mathrm{m} \times 3.15 \mathrm{~m} \quad(5.67 \quad$ Sq. meters $)$ accommodating 21 plants per plot per replication, three rows with 7 plants per row with spacing of $60 \times 45 \mathrm{~cm}$. All the recommended cultural practices were followed. Five randomly selected equally competitive plants from each row in each replication were tagged for the purpose of recording the observations on 15 characters viz. plant height $(\mathrm{cm})$, number of primary branches per plant, days to first flowering, days to $50 \%$ flowering, days to first fruit harvest, days to last fruit harvest, number of fruits per plant, fruit length $(\mathrm{cm})$, fruit width $(\mathrm{cm})$, average fruit weight $(\mathrm{g})$, fruit yield per plant $(\mathrm{kg})$, ascorbic acid $(\mathrm{mg} / 100 \mathrm{~g})$, total soluble solids ( $\left.{ }^{\circ} \mathrm{Brix}\right)$, lycopene content $(\mathrm{mg} / 100 \mathrm{~g})$ and betacarotene $(\mathrm{mg} / 100 \mathrm{~g})$.

\section{Results and Discussion}

The correlation coefficient results on the present investigation on tomato germplasm revealed that the fruit yield per plant recorded positive and significant correlations with plant height $(0.3282 \mathrm{P}$, $0.3584 \mathrm{G})$, number of primary branches per plant $(0.5203 \mathrm{P}, 0.5710 \mathrm{G})$, days to last fruit harvest $(0.4839 \mathrm{P}, 0.5490 \mathrm{G})$, number of fruits per plant $(0.4737 \mathrm{P}, 0.4971 \mathrm{G})$, fruit length $(0.3425 \mathrm{P}, 0.3934 \mathrm{G})$, fruit width $(0.4074 \mathrm{P}, 0.4617 \mathrm{G})$, average fruit weight (0.4297P, 0.4608G), ascorbic acid (0.3712P, 0.3954G), total soluble solids (0.3041P, 0.3010G) and lycopene content $(0.0208 \mathrm{P}, 0.0301 \mathrm{G})$. It also registered negative and significant correlations with days to first fruit harvest $(-0.3180 \mathrm{P}$,$0.3977 \mathrm{G})$ and beta-carotene $(-0.3309 \mathrm{P}$, $0.3520 \mathrm{G})$. Plant yield is a very complex character dependent on a number of components. Therefore knowledge of magnitude of correlation of yield with its attributes is of immense practical utility and has profound significance in the field of crop improvement. Without bringing an improvement in the yield component characters, the genetic improvement in fruit yield is not possible. The results on character association indicated significant positive association yield with plant height, number of primary branches per plant, number of fruits per plant, days to last harvest, fruit length, fruit width, average fruit weight, ascorbic acid, total soluble solids and lycopene content which indicated that the adequate knowledge of interrelationship between fruit yield per plant and its component themselves is useful for selection and simultaneous improvement in these characters. All the possible genotypic and phenotypic correlation coefficient between fruit yield and quality components is given in Table 2. The present study discloses that in general, genotypic correlation coefficient were higher than their phenotypic ones. Similar finding were observed by Nagariya et al., (2015) Sudesh and Anita (2016) and Singh et al., (2018).

Path coefficient is the ratio of standard deviation of the effect due to a given cause (independent variable) to the total standard 
deviation of the effect (dependant variable). This technique, which aims to improve a dependent character like yield when the independent characters have a significant relation in desirable direction and positive direct or indirect effect through other component traits on the dependent characters, it is used in plant breeding programs. Plant height showed negligible direct positive effect on fruit yield per plant at phenotypic level (0.003P) and low negative direct effect at genotypic level $(-0.1041 \mathrm{G})$, number of primary branches per plant showed negligible direct positive effect on fruit yield per plant at genotypic level $(0.083 \mathrm{G})$ and low negative direct effect at phenotypic level $(-0.1211 \mathrm{P})$, days to first flowering recorded negligible and low positive direct effect on fruit yield per plant at phenotypic level and genotypic level $(0.0350 \mathrm{P}, 0.1309 \mathrm{G})$, days to $50 \%$ flowering showed negligible positive direct effect at genotypic level on fruit yield per plant at genotypic level $(0.029 \mathrm{G})$ and negligible negative direct effect at phenotypic level (-0.0184P).

Table.1 List of genotypes and their sources

\begin{tabular}{|l|l|l|}
\hline S.No. & Genotypes & \multicolumn{1}{|c|}{ Source } \\
\hline $\mathbf{1 .}$ & EC-615055 & NBPGR, Hyderabad \\
\hline $\mathbf{2 .}$ & EC-620463 & NBPGR, Hyderabad \\
\hline $\mathbf{3 .}$ & EC-620428 & NBPGR, Hyderabad \\
\hline $\mathbf{4 .}$ & AVTO-1219 & WVC, Taiwan, China \\
\hline $\mathbf{5 .}$ & EC-620378 & NBPGR, Hyderabad \\
\hline $\mathbf{6 .}$ & EC-620382 & NBPGR, Hyderabad \\
\hline $\mathbf{7 .}$ & EC-620389 & NBPGR, Hyderabad \\
\hline $\mathbf{8 .}$ & EC-620395 & NBPGR, Hyderabad \\
\hline $\mathbf{9 .}$ & EC-620406 & NBPGR, Hyderabad \\
\hline $\mathbf{1 0 .}$ & EC-620427 & NBPGR, Hyderabad \\
\hline $\mathbf{1 1 .}$ & EC-620394 & NBPGR, Hyderabad \\
\hline $\mathbf{1 2 .}$ & EC-620422 & NBPGR, Hyderabad \\
\hline $\mathbf{1 3}$. & EC-631369 & NBPGR, Hyderabad \\
\hline $\mathbf{1 4}$. & EC-631379 & NBPGR, Hyderabad \\
\hline $\mathbf{1 5}$ & EC-620503 & NBPGR, Hyderabad \\
\hline $\mathbf{1 6 .}$ & AVTO-9803 & WVC, Taiwan, China \\
\hline $\mathbf{1 7}$ & AVTO-9804 & WVC, Taiwan, China \\
\hline $\mathbf{1 8 .}$ & AVTO-1002 & WVC, Taiwan, China \\
\hline $\mathbf{1 9}$. & AVTO-0101 & WVC, Taiwan, China \\
\hline $\mathbf{2 0 .}$ & Pusa Ruby & IARI, New Delhi \\
\hline $\mathbf{2 1 .}$ & PKM-1 & Periyakulam, TNAU \\
\hline $\mathbf{2 2 .}$ & Pant bahar & GBPUAT, Uttarakhand \\
\hline $\mathbf{2 3 .}$ & Arkavikas & IIHR, Bengaluru \\
\hline & & \\
\hline
\end{tabular}


Table.2 Phenotypic (P) and genotypic (G) correlation coefficients among yield and yield attributes in twenty three genotypes of tomato

\begin{tabular}{|c|c|c|c|c|c|c|c|c|c|c|c|c|c|c|c|c|}
\hline Characters & & $\begin{array}{l}\text { Plant } \\
\text { height } \\
(\mathrm{cm})\end{array}$ & $\begin{array}{l}\text { No. of } \\
\text { primary } \\
\text { branches } \\
\text { per plant }\end{array}$ & $\begin{array}{l}\text { Days to } \\
\text { first } \\
\text { flowering }\end{array}$ & $\begin{array}{l}\text { Days to } 50 \\
\% \\
\text { flowering }\end{array}$ & $\begin{array}{l}\text { Days to } \\
\text { first } \\
\text { fruit } \\
\text { harvest }\end{array}$ & $\begin{array}{l}\text { Days to } \\
\text { last fruit } \\
\text { harvest }\end{array}$ & $\begin{array}{l}\text { No. of } \\
\text { fruits } \\
\text { per } \\
\text { plant } \\
\end{array}$ & $\begin{array}{l}\text { Fruit } \\
\text { length }\end{array}$ & $\begin{array}{l}\text { Plant } \\
\text { height } \\
(\mathrm{cm})\end{array}$ & $\begin{array}{l}\text { No. of } \\
\text { primary } \\
\text { branches } \\
\text { per plant }\end{array}$ & $\begin{array}{l}\text { Days to } \\
\text { first } \\
\text { flowering }\end{array}$ & $\begin{array}{l}\text { Days to } 50 \\
\% \\
\text { flowering }\end{array}$ & $\begin{array}{l}\text { Days to } \\
\text { first } \\
\text { fruit } \\
\text { harvest }\end{array}$ & $\begin{array}{l}\text { Days to } \\
\text { last fruit } \\
\text { harvest }\end{array}$ & $\begin{array}{l}\text { No. of } \\
\text { fruits per } \\
\text { plant }\end{array}$ \\
\hline \multirow[t]{2}{*}{ Plant height (cm) } & $\mathbf{P}$ & 1.0000 & $0.7078 * *$ & -0.0967 & 0.0605 & 0.0104 & $0.3366 * *$ & $0.6517 * *$ & -0.2031 & -0.1577 & $-0.3265 * *$ & 0.2192 & $0.5101 * *$ & $0.2461^{*}$ & $-0.2380 *$ & $0.3282 *$ \\
\hline & G & 1.0000 & $0.7562 * *$ & -0.1068 & 0.1240 & 0.0034 & $0.3975^{* *}$ & $0.6908 * *$ & -0.2201 & -0.1729 & $-0.3493^{*} *$ & 0.2386 & $0.5506 * *$ & $0.2291 *$ & $-0.2503 *$ & $0.3584 *$ \\
\hline \multirow{2}{*}{$\begin{array}{l}\text { No. of primary } \\
\text { branches per plant }\end{array}$} & $\mathbf{P}$ & & 1.0000 & $-0.2892 *$ & -0.1242 & -0.2098 & $.0 .6673 * *$ & $0.7343 * *$ & -0.0831 & -0.0802 & -0.1760 & $0.3996 * *$ & $0.5451 * *$ & 0.1296 & -0.0271 & $0.5203 * *$ \\
\hline & G & & 1.0000 & $-0.3491^{*}$ & -0.1908 & -0.2379 & $0.7356^{* *}$ & $0.7598 * *$ & -0.0966 & -0.0724 & -0.2004 & $0.4228 * *$ & $0.5841 * *$ & 0.1288 & -0.0487 & $0.5710 * *$ \\
\hline \multirow{2}{*}{$\begin{array}{l}\text { Days to first } \\
\text { flowering }\end{array}$} & $\mathbf{P}$ & & & 1.0000 & $0.6009 * *$ & $0.5154 * *$ & $-0.4514 * *$ & $-0.4194 * *$ & $0.3650 * *$ & $0.2475 *$ & $0.2990^{*}$ & $0.2709 *$ & -0.1314 & $-0.4307 * *$ & -0.0627 & -0.0565 \\
\hline & G & & & 1.0000 & $0.8356 * *$ & $0.7363 * *$ & $-0.5751 * *$ & $-0.5094 * *$ & $0.4403 * *$ & $0.3377 *$ & $0.3836 *$ & $0.3029^{*}$ & -0.1617 & $-0.5335^{* *}$ & -0.1269 & -0.0750 \\
\hline \multirow{2}{*}{$\begin{array}{c}\text { Days to } 50 \% \\
\text { flowering }\end{array}$} & $\mathbf{P}$ & & & & 1.0000 & $0.3834 * *$ & $-0.4029 * *$ & $-0.2555 *$ & $0.3641 * *$ & 0.1530 & $0.3167 * *$ & 0.0767 & 0.0182 & $-0.2867 *$ & -0.1023 & 0.0529 \\
\hline & G & & & & 1.0000 & $0.7305 * *$ & $-0.6066^{* *}$ & $-0.3539 *$ & $0.4163 * *$ & 0.2462 & $0.4256^{*} *$ & 0.1666 & 0.0402 & -0.4111 & -0.1279 & 0.0920 \\
\hline \multirow{2}{*}{$\begin{array}{l}\text { Days to first fruit } \\
\text { harvest }\end{array}$} & $\mathbf{P}$ & & & & & 1.0000 & $-0.3044 *$ & $-0.2902 *$ & -0.0185 & -0.0562 & -0.1017 & 0.1567 & -0.0271 & $-0.2485^{*}$ & 0.0095 & $-0.3180^{*}$ \\
\hline & G & & & & & 1.0000 & -0.4056 & -0.3427 & -0.0555 & -0.0128 & -0.0880 & 0.1135 & -0.0067 & $-0.3016^{*}$ & 0.0162 & $-0.3977^{*}$ \\
\hline \multirow{2}{*}{$\begin{array}{l}\text { Days to last fruit } \\
\text { harvest }\end{array}$} & $\mathbf{P}$ & & & & & & 1.0000 & $0.6657 * *$ & $-0.2545^{*}$ & -0.0954 & -0.2254 & 0.1616 & $0.3226 * *$ & $0.2461 *$ & $-0.2478 *$ & $0.4839 * *$ \\
\hline & G & & & & & & 1.0000 & $0.7502 * *$ & $-0.2912^{*}$ & -0.0841 & -0.2581 & 0.1694 & $0.3518 * *$ & $0.2844 *$ & $-0.3059 *$ & $0.5490 * * *$ \\
\hline \multirow{2}{*}{$\begin{array}{l}\text { No of fruits } \\
\text { per plant }\end{array}$} & $\mathbf{P}$ & & & & & & & 1.0000 & $0.4545 * *$ & $0.4027 * *$ & $-0.5187 * *$ & $0.2824 *$ & $0.4904 * *$ & $0.4143 * *$ & $0.3452 * *$ & $0.4737 * *$ \\
\hline & G & & & & & & & 1.0000 & $-0.4880^{* * *}$ & $-0.4535 * *$ & $-0.5484 * *$ & $0.2954 *$ & $0.5012 * *$ & $0.4283 * *$ & $0.3615 * *$ & $0.4971 * *$ \\
\hline \multirow{2}{*}{$\begin{array}{l}\text { Fruit length } \\
\quad(\mathrm{cm})\end{array}$} & $\mathbf{P}$ & & & & & & & & 1.0000 & $0.7084 * *$ & $0.7615 * *$ & 0.2276 & 0.0844 & $-0.3723 * *$ & 0.1304 & $0.3425^{*}$ \\
\hline & $\mathbf{G}$ & & & & & & & & 1.0000 & $0.7927 * *$ & $0.8879 * *$ & 0.2430 & -0.0756 & $-0.4090 * *$ & 0.1417 & $0.3934 *$ \\
\hline \multirow{2}{*}{$\begin{array}{c}\text { Fruit width } \\
\text { (cm) }\end{array}$} & $\mathbf{P}$ & & & & & & & & & 1.0000 & $0.7324 * *$ & 0.0805 & -0.1527 & $-0.2816^{*}$ & -0.0341 & $0.4074 * *$ \\
\hline & G & & & & & & & & & 1.0000 & $0.8571 * *$ & 0.1015 & -0.1908 & $-0.3157^{*}$ & -0.0242 & $0.4617 * *$ \\
\hline \multirow{2}{*}{$\begin{array}{l}\text { Average fruit } \\
\text { weight }\end{array}$} & $\mathbf{P}$ & & & & & & & & & & 1.0000 & 0.0433 & -0.1971 & $-0.4212 * *$ & 0.1315 & $0.4297 * *$ \\
\hline & G & & & & & & & & & & 1.0000 & 0.0626 & -0.2173 & $-0.4574 * *$ & 0.1408 & $0.4608 * *$ \\
\hline \multirow{2}{*}{$\begin{array}{c}\text { Ascorbic acid } \\
\text { content } \\
(\mathbf{m g} / 100 \mathrm{~g})\end{array}$} & $\mathbf{P}$ & & & & & & & & & & & 1.0000 & 0.1145 & $-0.4182 * *$ & -0.0573 & $0.3712 * *$ \\
\hline & G & & & & & & & & & & & 1.0000 & 0.1185 & $-0.4482 * *$ & -0.0729 & $0.3954 * *$ \\
\hline \multirow[t]{2}{*}{ TSS ( ${ }^{0}$ Brix) } & $\mathbf{P}$ & & & & & & & & & & & & 1.0000 & $0.4243 * *$ & 0.1713 & $0.3041^{*}$ \\
\hline & G & & & & & & & & & & & & 1.0000 & $0.4481 * *$ & 0.1739 & $0.3010^{*}$ \\
\hline \multirow{2}{*}{$\begin{array}{l}\text { Lycopene content } \\
\quad(\mathrm{mg} / \mathbf{1 0 0 g})\end{array}$} & $\mathbf{P}$ & & & & & & & & & & & & & 1.0000 & -0.0684 & 0.0208 \\
\hline & G & & & & & & & & & & & & & 1.0000 & -0.802 & 0.0301 \\
\hline \multirow{2}{*}{$\begin{array}{l}\text { Beta-carotene } \\
\text { mg/100g) }\end{array}$} & $\mathbf{P}$ & & & & & & & & & & & & & & 1.0000 & $-0.3309 * *$ \\
\hline & G & & & & & & & & & & & & & & 1.0000 & $-0.3520 * *$ \\
\hline \multirow{2}{*}{$\begin{array}{l}\text { Fruit yield per } \\
\text { plant }(\mathrm{Kg})\end{array}$} & $\mathbf{P}$ & & & & & & & & & & & & & & & 1.0000 \\
\hline & G & & & & & & & & & & & & & & & 1.0000 \\
\hline
\end{tabular}


Table.3 Phenotypic (P) and genotypic (G) path coefficients indicating direct and indirect effects of components characters on fruit yield in twenty three genotypes of tomato

\begin{tabular}{|c|c|c|c|c|c|c|c|c|c|c|c|c|c|c|c|c|}
\hline Characters & & $\begin{array}{l}\text { Plant height } \\
\text { (cm) }\end{array}$ & $\begin{array}{c}\text { No. of } \\
\text { primary } \\
\text { branches } \\
\text { per plant }\end{array}$ & $\begin{array}{l}\text { Days to first } \\
\text { flowering }\end{array}$ & $\begin{array}{c}\text { Days to } 50 \\
\% \\
\text { flowering }\end{array}$ & $\begin{array}{l}\text { Days to first } \\
\text { fruit harvest }\end{array}$ & $\begin{array}{c}\text { Days to } \\
\text { last } \\
\text { fruit } \\
\text { harvest }\end{array}$ & $\begin{array}{c}\text { No. of } \\
\text { fruits } \\
\text { per } \\
\text { plant }\end{array}$ & $\begin{array}{c}\text { Fruit } \\
\text { length } \\
(\mathbf{c m})\end{array}$ & $\begin{array}{l}\text { Fruit width } \\
\quad(\mathrm{cm})\end{array}$ & $\begin{array}{c}\text { Average } \\
\text { fruit } \\
\text { weight(g } \\
\text { ) }\end{array}$ & $\begin{array}{l}\text { Ascorbic } \\
\text { acid } \\
\text { content } \\
\text { (mg/100g) }\end{array}$ & $\begin{array}{c}\text { TSS } \\
\left({ }^{0} \mathrm{Brix}\right)\end{array}$ & $\begin{array}{c}\text { Lycopene } \\
\text { content } \\
(\mathrm{mg} / 100 \mathrm{~g})\end{array}$ & $\begin{array}{l}\text { Beta -carotene } \\
\text { (mg/100g) }\end{array}$ & $\begin{array}{c}\text { Correlation } \\
\text { coefficient }\end{array}$ \\
\hline \multirow{2}{*}{ Plant height (cm) } & $\mathbf{P}$ & $\underline{0.0033}$ & 0.0023 & -0.0003 & 0.0002 & 0.0000 & 0.0011 & 0.0021 & -0.0007 & -0.0005 & -0.0011 & 0.0007 & 0.0017 & 0.0008 & -0.0008 & 0.0011 \\
\hline & G & $\underline{-0.1041}$ & -0.0787 & 0.0111 & -0.0129 & -0.0004 & -0.0414 & -0.0719 & 0.0229 & 0.0180 & 0.0364 & -0.0248 & -0.0573 & -0.0239 & 0.0261 & -0.0373 \\
\hline \multirow{2}{*}{$\begin{array}{c}\text { No. of primary } \\
\text { branches per plant }\end{array}$} & $\mathbf{P}$ & -0.0857 & $\underline{-0.1211}$ & 0.0350 & 0.0150 & 0.0254 & -0.0808 & -0.0889 & 0.0101 & 0.0097 & 0.0217 & -0.0484 & -0.0660 & -0.0157 & 0.0033 & -0.0630 \\
\hline & G & 0.0629 & $\underline{0.0832}$ & -0.0290 & -0.0159 & -0.0198 & 0.0612 & 0.0632 & -0.0080 & -0.0060 & -0.0167 & 0.0352 & 0.0486 & 0.0107 & -0.0041 & 0.0475 \\
\hline \multirow{2}{*}{$\begin{array}{l}\text { Days to first } \\
\text { flowering }\end{array}$} & $\mathbf{P}$ & -0.0034 & -0.0101 & $\underline{\mathbf{0 . 0 3 5 0}}$ & 0.0210 & 0.0180 & -0.0158 & -0.0147 & & 0.0087 & 0.0105 & 0.0095 & -0.0046 & -0.0151 & -0.0022 & -0.0020 \\
\hline & G & -0.0140 & -0.0457 & $\underline{0.1309}$ & 0.1094 & 0.0964 & -0.0753 & -0.0667 & 0.0576 & 0.0442 & 0.0502 & 0.0397 & -0.0212 & --0.0698 & --0.0166 & -0.0098 \\
\hline \multirow{2}{*}{$\begin{array}{c}\text { Days to } 50 \% \\
\text { flowering }\end{array}$} & $\mathbf{P}$ & -0.0011 & 0.0023 & -0.0110 & -0.0184 & -0.0070 & 0.0074 & 0.0047 & -0.0067 & -0.0028 & -0.0058 & -0.0014 & -0.0003 & 0.0053 & 0.0019 & -0.0010 \\
\hline & G & 0.0066 & -0.0101 & 0.0442 & $\underline{0.0529}$ & 0.0387 & -0.0321 & -0.0187 & 0.0220 & 0.0130 & 0.0225 & 0.0088 & 0.0021 & -0.0218 & -0.0068 & 0.0049 \\
\hline \multirow{2}{*}{$\begin{array}{c}\text { Days to first fruit } \\
\text { harvest }\end{array}$} & $\mathbf{P}$ & 0.0003 & -0.0054 & 0.0132 & 0.0098 & $\underline{0.0257}$ & -0.0078 & -0.0075 & -0.0005 & -0.0014 & -0.0026 & 0.0040 & -0.0007 & -0.0064 & 0.0002 & -0.0082 \\
\hline & G & -0.0002 & 0.0136 & -0.0420 & -0.0416 & $\underline{-0.0570}$ & 0.0231 & 0.0195 & 0.0032 & 0.0007 & 0.0050 & -0.0065 & 0.0004 & 0.0172 & -0.0009 & 0.0227 \\
\hline \multirow{2}{*}{$\begin{array}{c}\text { Days to last fruit } \\
\text { harvest }\end{array}$} & $\mathbf{P}$ & 0.0463 & 0.0918 & -0.0621 & -0.0554 & -0.0419 & $\underline{0.1375}$ & 0.0915 & -0.0350 & -0.0131 & -0.0310 & 0.0222 & 0.0444 & 0.0338 & -0.0341 & 0.0665 \\
\hline & G & 0.0144 & 0.0267 & -0.0209 & 0.0220 & -0.0147 & $\underline{0.0363}$ & 0.0272 & -0.0106 & -0.0031 & -0.0094 & 0.0062 & 0.0128 & 0.0103 & -0.0111 & \begin{tabular}{|l|}
0.0199 \\
\end{tabular} \\
\hline \multirow[t]{2}{*}{$\begin{array}{l}\text { No of fruits } \\
\text { per plant }\end{array}$} & $\mathbf{P}$ & 0.5608 & 0.6319 & -0.3609 & -0.2199 & -0.2498 & 0.5728 & $\underline{0.8606}$ & -0.3912 & -0.3466 & -0.4464 & 0.2430 & 0.4220 & 0.3565 & -0.2970 & 0.4076 \\
\hline & G & 0.6313 & 0.6944 & -0.4656 & -0.3235 & -0.3132 & 0.6856 & $\underline{0.9139}$ & -0.4460 & -0.4144 & -0.5012 & 0.2699 & 0.4581 & 0.3914 & -0.3304 & 0.4543 \\
\hline \multirow{2}{*}{$\begin{array}{l}\text { Fruit length } \\
\quad(\mathrm{cm})\end{array}$} & $\mathbf{P}$ & -0.0206 & -0.0084 & 0.0370 & 0.0369 & -0.0019 & -0.0258 & -0.0461 & $\underline{0.1014}$ & 0.0719 & 0.0772 & 0.0231 & -0.0086 & -0.0378 & 0.0132 & 0.0347 \\
\hline & G & 0.0516 & 0.0226 & -0.1032 & -0.0976 & 0.0130 & 0.0682 & 0.1144 & $\underline{-0.2343}$ & -0.1858 & -0.2081 & -0.0570 & 0.0177 & 0.0958 & -0.0332 & -0.0922 \\
\hline \multirow{2}{*}{$\begin{array}{l}\text { Fruit width } \\
\quad(\mathbf{c m})\end{array}$} & $\mathbf{P}$ & -0.0242 & -0.0123 & 0.0380 & 0.0235 & -0.0086 & -0.0147 & -0.0619 & 0.1088 & $\underline{0.1536}$ & 0.1125 & 0.0124 & -0.0235 & -0.0433 & -0.0052 & 0.0626 \\
\hline & G & -0.0215 & -0.0090 & 0.0419 & 0.0306 & -0.0016 & -0.0104 & -0.0563 & 0.0984 & $\underline{0.1242}$ & 0.1064 & 0.0126 & -0.0237 & -0.0392 & -0.0030 & 0.0573 \\
\hline \multirow{2}{*}{$\begin{array}{l}\text { Average fruit } \\
\text { weight }\end{array}$} & $\mathbf{P}$ & -0.2420 & -0.1327 & 0.2216 & 0.2347 & -0.0754 & -0.1670 & -0.3844 & 0.5643 & 0.5428 & $\underline{0.7411}$ & 0.0321 & -0.1461 & -0.3122 & 0.0975 & 0.3184 \\
\hline & G & -0.3743 & -0.2147 & 0.4111 & 0.4561 & -0.0943 & -0.2766 & -0.5878 & 0.9516 & 0.9186 & $\underline{1.0717}$ & 0.0671 & -0.2329 & -0.4902 & 0.1509 & 0.4938 \\
\hline \multirow{2}{*}{$\begin{array}{c}\text { Ascorbic acid } \\
\text { content } \\
(\mathbf{m g} / \mathbf{1 0 0 g})\end{array}$} & $\mathbf{P}$ & 0.0173 & 0.0315 & 0.0214 & 0.0060 & 0.0124 & 0.0127 & 0.0223 & 0.0179 & 0.0063 & 0.0034 & $\underline{0.0788}$ & 0.0090 & -0.0330 & -0.0045 & 0.0293 \\
\hline & G & 0.0283 & 0.0502 & 0.0360 & 0.0198 & 0.0135 & 0.0201 & 0.0351 & 0.0289 & 0.0120 & 0.0074 & $\underline{0.1188}$ & 0.0141 & -0.0532 & -0.0087 & 0.0470 \\
\hline \multirow[t]{2}{*}{ TSS ( ${ }^{0}$ Brix) } & $\mathbf{P}$ & 0.0387 & 0.0413 & -0.0100 & 0.0014 & -0.0021 & 0.0245 & 0.0372 & -0.0064 & -0.0116 & -0.0149 & 0.0087 & $\underline{0.0758}$ & 0.0322 & 0.0130 & 0.0231 \\
\hline & G & 0.0089 & 0.0095 & -0.0026 & 0.0007 & -0.0001 & 0.0057 & 0.0081 & -0.0012 & -0.0031 & -0.0035 & 0.0019 & $\underline{0.0162}$ & 0.0073 & 0.0028 & 0.0049 \\
\hline \multirow{2}{*}{$\begin{array}{l}\text { Lycopene content } \\
\quad(\mathrm{mg} / \mathbf{1 0 0 g})\end{array}$} & $\mathbf{P}$ & 0.0118 & 0.0062 & -0.0206 & -0.0137 & -0.0119 & 0.0118 & 0.0198 & -0.0178 & -0.0135 & -0.0201 & -0.0200 & 0.0203 & $\underline{0.0478}$ & -0.0033 & 0.0010 \\
\hline & G & 0.0429 & 0.0241 & 0.0999 & 0.0770 & 0.0585 & 0.0533 & 0.0802 & 0.0766 & 0.0591 & 0.0857 & 0.0839 & 0.0839 & $\underline{0.1873}$ & 0.0150 & 0.0056 \\
\hline \multirow{2}{*}{$\begin{array}{c}\text { Beta-carotene } \\
\mathrm{mg} / 100 \mathrm{~g})\end{array}$} & $\mathbf{P}$ & 0.0269 & 0.0031 & 0.0071 & 0.0115 & -0.0011 & 0.0280 & 0.0390 & -0.0147 & 0.0039 & -0.0148 & 0.0065 & -0.0193 & 0.0077 & $-\underline{0.1129}$ & 0.0373 \\
\hline & G & 0.0255 & 0.0050 & 0.0130 & 0.0131 & -0.0017 & 0.0312 & 0.0369 & -0.0145 & 0.0025 & -0.0144 & 0.0074 & -0.0177 & 0.0082 & $-\underline{-0.1021}$ & 0.0359 \\
\hline
\end{tabular}

Phenotypic Residual effect $=0.304$; Genotypic Residual effect= 0.233; Diagonal (under lined) values indicate direct effect 
Days to first fruit harvest showed negligible positive direct effect on fruit yield per plant was exhibited by this trait at phenotypic level $(0.0257 \mathrm{P})$ and negligible negative direct effect at genotypic level $(-0.057 \mathrm{G})$. Days to last fruit harvest showed low and negligible positive direct effect on fruit yield per plant at phenotypic level and genotypic level $(0.1375 \mathrm{P}, 0.0363 \mathrm{G})$, number of fruits per plant showed at both phenotypic and genotypic level, fruit yield per plant showed high positive direct effect $(0.8606 \mathrm{P}, 0.9139 \mathrm{G})$ on fruit yield per plant respectively. Fruit length $(\mathrm{cm})$ showed low positive on fruit yield per plant (0.1014P) at phenotypic level and negligible negative direct effects at genotypic level $(-0.2343 G)$. Fruit width showed low positive direct effect on fruit yield per plant both at phenotypic and genotypic (0.1536P, $0.1242 \mathrm{G}$ ) levels, Average fruit weight showed moderate and high positive direct effect on fruit yield per plant both at phenotypic and genotypic $(0.7411 \mathrm{P}, 1.0717 \mathrm{G})$ levels (Table 3).

Ascorbic acid content (mg/100g) showed negligible and low positive direct effect on fruit yield per plant both at phenotypic and genotypic $(0.0788 \mathrm{P}, 0.1188 \mathrm{G})$ levels. Total soluble solids ( ${ }^{\circ}$ Brix) showed negligible positive direct effect on fruit yield per plant both at phenotypic and genotypic $(0.0758 \mathrm{P}$, $0.0162 \mathrm{G})$ levels. Lycopene content recorded negligible direct effect on fruit yield per plant both at phenotypic (0.0478P) and low genotypic $(0.1873 \mathrm{G})$ levels. Beta-carotene recorded negligible negative direct effects on fruit yield per plant at genotypic level ($0.1129 \mathrm{P})$ and phenotypic level (-0.1021G) respectively. The traits like number of fruits per plant and average fruit weight exhibited positive direct effects on fruit yield and these traits also recorded positive correlation with yield. This suggested that direct selection based on these traits will be rewarding for crop yield improvement. Similar results were also reported in tomato by Asati et al., (2008), Tiwari et al., (2013), Meitei et al., (2014) and Rakesh et al., (2014). In this study, it can be concluded that days to last fruit harvest, number of fruits per plant, fruit width, average fruit weight, ascorbic acid, total soluble solids, lycopene content and betacarotene showed positive correlation and positive direct effect on fruit yield per plant. These are identified as superior yield components. Hence, the genotypes which exhibited better performance for these characters can be used in further improvement of tomato.

The residual factor determines how best the causal factors account for the variability of the dependent factor, the fruit yield per plant in this case. The residual effects were 0.304 and 0.233 , which were of low magnitude at genotypic and phenotypic levels.

Hence, it could be concluded that in tomato yield per plant was positively and significantly correlated with plant height, number of primary branches per plant, number of fruits per plant, days to last harvest, fruit length, fruit width, average fruit weight, ascorbic acid, total soluble solids and lycopene content. In path coefficient analysis the highest positive direct effect was noted in average fruit weight, followed by number of fruits per plant. So, the traits like average fruit weight and number of fruits per plant showed positive correlation with yield as well as they have direct effect on yield. Hence these traits can be used for selection in tomato to bring about the improvement in yield.

\section{References}

Asati, B. S., Rai, N. and Singh, A. K. 2008. Genetic parameters study for yield and quality traits in tomato Asian J. Horti., 3(2): 222-225.

Giovannucci, E., Rimm, E. B., Liu, Y., 
Stamper M. J. and Willett, W. C.2002. A prospective study of tomato products, lycopene, and prostate cancer risk. J. Natl. Cancer Inst., 94 (5): 391-398.

Islam, B. M. R., Ivy, N. A., Rasul, M. G. and Zakaria, M. 2010. Character association and path analysis of exotic tomato (Solanum lycopersicum L.) genotypes. Bangladesh. J. Plant Breed. Genet., 23 (1):13-18.

Meitei, K. M., Broda, G. C., Singh, S. J. and Sinha, A. K. 2014. Morphology based genetic variability analysis and identification of important characters for tomato (Solanum lycopersicum L.). Am. Eur. J. Agril. Envi Sci., 14(10): 1105- 1111.

Nagariya, N. K., Bhardwaj, R., Sharma, N., Mukherjee, S. and Umesh. 2015. Correlation and path analysis in tomato (Solanum lycopersicon L.). Int. J. Farm Sci., 5(4): 111-117.

Rakesh, K., Ram, C. N., Yadav, G. C., Chandra, D., Vimal, S. C. and Bhartiya,
H. D. 2014. Studies on correlation and path coefficient analysis in tomato (Solanum lycopersicum). Plant Archives., 14: 443-447.

Singh, A, K., Solankey, S. S., Akhtar, S., Kumari, P. and Chaurasiya, J. 2018. Correlation and Path Coefficient Analysis in Tomato (Solanum lycopersicum L.). Int. J. Curr. Microbiol. App. Sci., 7: 4278-4285.

Sudesh, K. and Anita, S. 2016. Assessment of genetic variability, character association and path analysis in tomato (Solanum lycopersicum L.) under tarai condition of Uttarakhand. Int. J. Agril. Sci., 34(8): 1706-1709.

Tiwari, J. K., Tiwari, A. K. and Mehta, N. 2013. Selection strategies for fruit yield in tomato (Solanum lycopersicum L.). Vegetable Science. 40(1): 23-27.

Vavilov, N. I. 1951. The origin, variation, immunity and breeding of cultivated plants. Chronica Botany. 13: 1-366.

\section{How to cite this article:}

Sushma, K., P. Saidaiah, K. Ravinder Reddy, Harikishan Sudini and Geetha, A. 2020. Correlation and Path Coefficient Analysis in Tomato (Solanum lycopersicum L.). Int.J.Curr.Microbiol.App.Sci. 9(11): 2569-2575. doi: https://doi.org/10.20546/ijcmas.2020.911.311 